\title{
Histoplasma endocarditis on a stenosed aortic valve presenting as dysphagia and weight loss
}

\author{
P T Wilmshurst, G E Venn, S J Eykyn
}

Department of
Cardiology
P T Wilmshurst
Department of
Cardiothoracic
Surgery
G E Venn
Department of
Microbiology,
St Thomas' Hospital,
London
S J Eykyn
Correspondence to:
Dr P T Wilmshurst,
Royal Infirmary,
Huddersield HD3.3EA.

\begin{abstract}
A 40-year-old man with aortic stenosis and disseminated histoplasmosis did not respond to treatment with itraconazole. Though there was no haemodynamic deterioration, valvar regurgitation, or embolic phenomena a presumptive diagnosis of infective endocarditis was made. This was confirmed at aortic valve replacement. Antifungal treatment was continued for 18 months after valve replacement and serological tests for Histoplasma became progressively more negative during a three year follow up.
\end{abstract}

(Br Heart f 1993;70:565-567)

Disseminated histoplasmosis is a rare and frequently fatal infection that sometimes involves the heart or great vessels, producing infective endocarditis or endarteritis. ${ }^{1}$ We are unaware of any previous reported case of Histoplasma endocarditis in the United Kingdom and report a patient with disseminated histoplasmosis and endocarditis who was successfully treated. He remained well for three years and histoplasma serology was measured throughout that period.

\section{Case report}

An unmarried heterosexual white 40-year-old man presented in March 1990 with a two month history of dysphagia, sore throat, and anorexia. He reported that he had lost 2 stones in weight and had a productive cough with green sputum. His symptoms had started while he was working on a cruise liner in the Caribbean and on the South East coast of the United States of America though he reported that he had not been ashore while in port. Initially he had been investigated in the United States and a biopsy specimen was taken of an ulcerated lesion on the epiglottis. Histological examination showed chronic inflammation. No cultures were performed. He returned to the United Kingdom for further investigation and treatment. $\mathrm{He}$ had been known to have a cardiac murmur since childhood and had previously been treated for alcohol abuse.

On admission he was cachectic and pale with a low grade fever $\left(37 \cdot 5-38^{\circ} \mathrm{C}\right)$. There was an aortic ejection systolic murmur but no evidence of valvar regurgitation or heart failure. There were no splinter haemorrhages, clubbing, or other features of infective endocarditis.
Investigations showed a normochromic normocytic anaemia (haemoglobin concentration $9.4 \mathrm{~g} / \mathrm{dl}$ ), neutropenia (white cell count $3.7 \times 10^{9} / 1$ with reduced granulocytes and lymphocytes), erythrocyte sedimentation rate $30 \mathrm{~mm} / \mathrm{h}$ (Westergren), C-reactive protein $71 \mathrm{mg} / \mathrm{l}$, and albumin $36 \mathrm{~g} / 1$. The plasma concentration of sodium was low (131 $\mathrm{mmol} / \mathrm{l})$ and potassium was high $(5 \cdot 2$ $\mathrm{mmol} / \mathrm{l})$. Renal and liver function were otherwise normal. Numerous blood cultures were sterile despite prolonged incubation. Sputum culture was negative for Mycobacterium tuberculosis and other pathogens. Another biopsy specimen was taken of the ulcer on the epiglottis.

The chest $x$ ray was normal. Electrocardiogram showed sinus rhythm with voltage criteria of left ventricular hypertrophy. Echocardiography showed concentric left ventricular hypertrophy with thickening and reduced opening of the aortic valve. Doppler examination suggested severe aortic stenosis but only trivial aortic regurgitation.

At cardiac catheterisation the aortic valve was found to be severely calcified with a peak gradient of $142 \mathrm{~mm} \mathrm{Hg}$ and mean gradient of $55 \mathrm{~mm} \mathrm{Hg}$. Cardiac output was $5 \cdot 21 / \mathrm{min}$ and the calculated Gorlin valve area was $0.9 \mathrm{~cm}^{2}$. There was mild aortic regurgitation but good left ventricular function (ejection fraction $57 \%$ ) and normal coronary arteries. During cardiac catheterisation the aortic valve was crossed retrogradely.

After cardiac catheterisation the patient's temperature rose to $39^{\circ} \mathrm{C}$ and he became toxic. Further blood cultures were taken but grew no organisms. Five days after cardiac catheterisation the biopsy specimens of the epiglottis were reported to show yeasts suggestive of Histoplasma. Cultures of the biopsy material grew scanty Candida albicans but were only incubated for $\mathbf{4 8}$ hours. Serological tests for Histoplasma were positive (table). A bone marrow biopsy specimen was histologically normal but Histoplasma capsulatum was isolated on culture after three weeks' incubation. A biopsy specimen of an ulcer on the foot which had appeared after admission showed a non-caseating granuloma but no organisms were isolated on culture.

A computer scan of the abdomen showed moderate splenomegaly and enlargement of both adrenal glands. A short Synacthen (tetracosactrin) test showed a borderline impaired response. Tests for HIV I and II antibodies were negative.

Disseminated histoplasmosis was diag- 
Results of Histoplasma serology

\begin{tabular}{|c|c|c|c|c|}
\hline \multirow[b]{2}{*}{ Date } & \multicolumn{2}{|c|}{ Complement fixation tests } & \multicolumn{2}{|c|}{ Immunodiffusion } \\
\hline & Mycelial antigen & Yeast antigen & $H$ line & $M$ line \\
\hline $\begin{array}{l}18 \text { April } 1990 \\
21 \text { May } 1990 \\
26 \text { June } 1990 \\
12 \text { July } 1990 \\
21 \text { Sept } 1990 \\
26 \text { Oct } 1990 \\
21 \text { Dec } 1990 \\
12 \text { Feb } 1991 \\
9 \text { April } 1991 \\
2 \text { July } 1991 \\
2 \text { Oct } 1991 \\
8 \text { Jan } 1992 \\
8 \text { April } 1992 \\
5 \text { Oct } 1992\end{array}$ & $\begin{array}{l}1: 640 \\
1: 640 \\
1: 320 \\
1: 320 \\
1: 320 \\
1: 320 \\
1: 160 \\
1: 80 \\
1: 80 \\
1: 40 \\
1: 20 \\
1: 20 \\
1: 10 \\
\text { Negative }\end{array}$ & $\begin{array}{l}1: 640 \\
1: 640 \\
1: 320 \\
1: 160 \\
1: 160 \\
1: 320 \\
1: 320 \\
1: 40 \\
1: 40 \\
1: 40 \\
1: 20 \\
1: 20 \\
1: 10 \\
\text { Negative }\end{array}$ & $\begin{array}{l}+ \\
+ \\
+ \\
+ \\
+ \\
+ \\
+ \\
+ \\
\text { Weak }+ \\
\text { Weak }+ \\
- \\
- \\
\text { Reported } \\
-\end{array}$ & $\begin{array}{l}+ \\
+ \\
+ \\
+ \\
+ \\
+ \\
+ \\
+ \\
+ \\
+ \\
\text { Weak }+ \\
\text { Weak }+ \\
\text { "Positive" } \\
-\end{array}$ \\
\hline
\end{tabular}

nosed and treatment with oral itraconazole (200 mg daily) was started. During the next month, the epiglottic lesion regressed but he continued to have spikes of fever and despite transfusion with a total of nine units of blood his haemoglobin concentration fell progressively to $7 \cdot 1 \mathrm{~g} / \mathrm{dl}$ though there was no evidence of haemolysis. There was no haemodynamic deterioration or any emboli, but it was thought that his failure to improve with itraconazole was probably the result of endocarditis on the aortic valve.

Treatment with intravenous amphotericin (in gradually increasing doses to a maximum $50 \mathrm{mg}$ daily) was started four days before aortic valve replacement on 25 May 1990. At operation the aortic valve had massive friable vegetations and histological examination showed it to be fibrosed, heavily calcified, and inflamed with numerous fungal colonies, consistent with Histoplasma. $H$ capsulatum was isolated on culture. A Carbomedic Bileaflet $(23 \mathrm{~mm})$ valve was inserted.

By the ninth postoperative day the patient was apyrexial, fully mobile, and the epiglottic lesion had completely healed. The only postoperative problem was the development of a large ( $1.8 \mathrm{l}$ ) bloodstained pericardial effusion, which was drained percutaneously. Intravenous amphotericin ( $50 \mathrm{mg}$ daily) was discontinued 50 days after operation because of deteriorating renal function, after a total dose of $2.55 \mathrm{~g}$. On the 51 postoperative day treatment with itraconazole (400 mg daily) was started. This continued for 17 months (until December 1991). He was discharged home on the 58 postoperative day. Haemoglobin concentration and white cell count gradually increased but they did not return to the normal range until one year after the operation, by which time he had regained his lost weight. Tests of adrenal function in December 1990 were entirely normal. During the three years since valve replacement Histoplasma serology became negative (table).

\section{Discussion}

Histoplasmosis is usually a benign infection. It is common in the United States of America and the Caribbean, where this patient probably became infected. He showed many of the features typical of the rare and more seri- ous form of disseminated histoplasmosis, in which systemic features such as weight loss, fever, and malaise are almost universal. ${ }^{1}$ Anaemia, leucopenia, and splenomegaly are also common and suggest involvement of marrow and lymphoid tissue, which if cultured often allows isolation of the organism, as in this case. ${ }^{12}$ Ulceration of the upper gastrointestinal and respiratory tract is present in over $25 \%$ of cases and again biopsy and culture are often diagnostic. ${ }^{2}$ Oropharyngeal and laryngeal lesions are more common in chronic disseminated disease than in acute disseminated infection, and ulceration of the tongue and buccal mucosa is more common than ulceration of the epiglottis. ${ }^{1}$ Biopsy of the skin lesions, when present, will also often yield a diagnosis. ${ }^{2}$ Addison's disease caused by adrenal involvement is common. ${ }^{12}$ Infection of the central nervous and cardiovascular systems is often associated with a fatal outcome. ${ }^{134}$

Histoplasma endocarditis can affect apparently normal cardiac valves as well as grossly abnormal structures such as diseased and prosthetic valves and cardiac tumours. ${ }^{4}$ Infection of atheroma and aneurysms in great vessels and vascular grafts has also been described. ${ }^{45}$ The main consequence of such endocardial and endothelial involvement is systemic embolisation, frequently with massive emboli, but often there is little haemodynamic deterioration or valvar regurgitation. ${ }^{45}$

$H$ capsulatum is a slow growing dimorphic fungus and is rarely grown from blood cultures even in patients with endocarditis, though positive blood cultures have been reported in patients with progressive disseminated histoplasmosis in the absence of endocarditis. ${ }^{2}$

Disseminated histoplasmosisis is more common in males than females and in those who are immunocompromised. ${ }^{1}$ Alcohol abuse may predispose to disseminated infection and alcoholism is known to reduce the host response to several infective organisms. ${ }^{5}$

The usual treatment for disseminated histoplasmosis with endocarditis includes amphotericin. The total dose required is unknown, but in the only reported case of survival without surgery the patient received a total of $4.8 \mathrm{~g}$ amphotericin. ${ }^{6}$ All other patients who survived required valve replacement and the total dose of amphotericin ranged from $2.0 \mathrm{~g}$ to $3.4 \mathrm{~g}$. Our patient had been treated with $2.55 \mathrm{~g}$ amphotericin before deteriorating renal function necessitated a change to itraconazole, which was continued for 17 months. Itraconazole had also been given for one month before valve replacement, but during that time there was evidence of disease progression. It is unlikely that any antifungal drug would have been successful without valve surgery.

In vitro studies showed that Histoplasma spp are very sensitive to itraconazole, which has greater in vitro potency than ketoconazole. $^{7}$ Both drugs have been used to treat local and disseminated histoplasmosis, but there has been concern about their use in 
severe and life-threatening histoplasmosis, particularly when there is central nervous system involvement. ${ }^{89}$ Though higher tissue concentrations are achieved with itraconazole than ketoconazole, both drugs penetrate the cerebrospinal fluid poorly. ${ }^{7}$ In limited in vivo studies itraconazole seemed to be as effective or more effective than ketoconazole for treating histoplasmosis and was better tolerated. ${ }^{8}$ We are unaware of reports of the use of either drug for treatment of histoplasma endocarditis.

Histoplasmosis can be chronic and relapsing so it is difficult to be sure that a cure has been achieved. Haematological and biochemical variables slowly improved in our patient but the most convincing evidence of a cure came from serial serological tests which became progressively negative (table). A positive complement fixation test with the yeast phase antigen is generally obtained with active acute disease, but it is also positive in most chronic cases. The mycelial phase antigen (histoplasmin) is helpful in detecting chronic disease. In the immunodiffusion test, the precipitin line closest to the serum wells is designated " $\mathrm{H}$ " because it is assumed that it indicates proven histoplasmosis. The line closer to the antigen well is designated " $M$ " because it is induced with a positive skin test using the mycelial antigen of $H$ capsulatum. The $\mathrm{H}$ band indicates active infection and the
$M$ band indicates either infection (acute or chronic) or a previous skin test to histoplasmin. The serological tests in this case suggest that the patient was cured.

Serological tests were performed at the Mycology Reference Laboratory, Public Health Service Laboratory, Colindale. We thank Professor R J Hay for helpful advice and we are grateful to our colleagues in the Ear, Nose and Throat Department and the Department of Histopathology for their help in management of this patient.

1 Goodwin RA, Shapiro JL, Thurman GH, Thurman SS, Des Prez RM. Disseminated histoplasmosis: clinical and pathological correlations. Medicine 1980;59:1-33.

2 Smith JW, Utz JP. Progressive disseminated histoplasmosis. A prospective study of 26 patients. Ann Intern Med 1972;76:557-65.

Bradsher RW, Alford RH, Hawkins SS, Spickard WA. Conditions associated with relapse of amphotericin Btreated disseminated histoplasmosis. fohn Hopkins Med f 1982;150:127-31.

4 Bradsher RW, Wickre CG, Savage AM, Harston WE, Alford RH. Histoplasma capsulatum endocarditis cured by amphotericin B combined with surgery. Chest 1980; 78:791-5.

5 Hawkins SS, Gregory DW, Alford RH. Progressive disseminated histoplasmosis: favorable response to ketoconazole. Ann Intern Med 1981;95:446-9.

6 Derby BM, Coolidge K, Rogers DE. Histoplasma capsulatum endocarditis with major arterial embolism. Arch tum endocarditis with major

7 Grant SM, Clissold SP. Itraconazole. A review of its pharmacodynamic and pharmacokinetic properties, and therapeutic use in superficial and systemic mycoses. Drugs 1989;37:310-44.

8 Dismukes WE, Bradsher RW, Cloud GC, Kauffman CA, Chapman SW, George RB, et al. Itraconazole therapy for blastomycosis and histoplasmosis. Am $\mathcal{F}$ Med 1992; 93:489-97.

9 Saag MS, Dismukes WE. Treatment of histoplasmosis and blastomycosis. Chest 1988;93:848-51. 